\title{
Ulvan as novel reducing and stabilizing agent from renewable algal biomasses: application to green synthesis of silver
}

nanoparticles.

$\underline{\text { Alessio Massironi }^{\wedge} 1}$, Andrea Morelli^${ }^{\wedge}$, Lucia Grassi ${ }^{2}$, Dario Puppi ${ }^{1}$, Simona Braccini ${ }^{1}$, Giuseppantonio Maisetta², Semih Esin², Giovanna Batoni², Cristina Della Pinac, Federica $\underline{\text { Chiellini }^{*}}$

1 Department of Chemistry and Industrial Chemistry, University of Pisa, UdR INSTM PISA, Via Giuseppe Moruzzi 13, 56124

2 Dep. of Translational Research and New Technologies in Medicine and Surgery, University of Pisa. c Department of Chemistry, University of Milano, ISTM-CNR, Milano, Italy

$\wedge$ These authors contributed equally to the work.

*Corresponding author at: Prof. Federica Chiellini Tel. +039 0502219333; Fax: +039 0502219260.

Email address: federica.chiellini@ unipi.it

\section{$\underline{\text { Abstract }}$}

Silver nanoparticles (AgNPs) have been intensively investigated in virtue of their optical and antimicrobial properties, although their applications have been limited due to inherent toxicity and to the need of employing harsh chemical reagents for the synthesis. In this work, ulvan, a sulfated polysaccharide extracted from green algae belonging to Ulva armoricana sp., was for the first time investigated and identified as reducing and stabilizing agent for AgNPs synthesis by using milder conditions than those conventionally adopted by chemical methods. The synthesized AgNPs were thoroughly characterized to highlight the structure and the role exerted by ulvan in their synthesis and stabilization. The formation of AgNPs stabilized by a thick ulvan shell was assessed by UV-vis, XRD, TEM, DLS and zeta potential analyses. The developed Ulvan based AgNps showed an IC50 in the range of $10 \mu \mathrm{g} / \mathrm{ml}$ in Balb/3T3 mouse embryo fibroblasts and antimicrobial activity toward both Gram+and Gram - bacteria.

Keywords: 
Ulvan, silver nanoparticle, green chemistry, antimicrobial activity

Highlights:

- Ulvan is an underexploited algal polysaccharide with promising properties for biomedical applications.

- AgNPs were unprecedentedly synthetized by using ulvan as stabilizing and reducing agent in mild condition.

- AgNPs colloidal suspension proved stable in simulated physiological conditions.

- AgNPs showed a fast bactericidal activity against models pathogenic bacteria. 


\section{Introduction}

Nanotechnology is a promising interdisciplinary field of research, which has been applied so far in a wide array of applications including medicine, food conservation, electronics, chemical sensors etc. A large variety of organic, inorganic and particularly hybrid nanostructures have been to date investigated thanks to their tremendous activity provided by the large surface area exposed at the interfaces. Metal nanoparticles have fascinated scientists for over a century resulting broadly investigated in many diverse applications (Franci et al., 2015; Qazi \& Javaid, 2016). Among noble metals, silver received a noticeable interest in virtue of its intrinsic antimicrobial activity (Kim et al., 2007). Silver nanoparticles (AgNPs) have been intensively investigated in a wide range of industrial applications spanning from food packaging (Carbone, Tommasa, \& Sabbatella, 2016), textile preservations (Zhang, Wu, Chen, \& Lin, 2009), medicine (Ge et al., 2014), cosmetic products preservation and pharmacy (Gajbhiye \& Sakharwade, 2016). The mechanism of action of AgNPs against pathogenic bacteria has not been yet well elucidated since it is a process which occur by means of several complex pathways (Prabhu \& Poulose, 2012). Indeed AgNPs are thought to penetrate the bacterial cell wall thus provoking structural changes of the cell membrane which leads to cell death by means of impaired cell permeability (Dakal, Kumar, Majumdar, \& Yadav, 2016). However, the most accepted theory relevant to the AgNPs antimicrobial activity relies on the controlled release of silver ions by the metal nanoparticles whose efficacy was found to be size and concentration dependent (Dakal et al., 2016). Accordingly Ag ions were hypothesized to display their activity by two different processes: a) as catalyst for the production of oxygen radicals that oxidize molecular structures of bacteria, b) by means of electrostatic interactions with the negative charges of the bacterial cell membrane (Wang, Hu, \& Shao, 2017). The procedure followed for AgNPs fabrication strongly affects the properties of the synthesized nanoparticles and ultimately their fields of applications Nguyen et al., 2013; Raveendran, Fu, \& Wallen, 2003). Strong reducing agents of synthetic origins, such as sodium borohydride ( $\mathrm{NaBH} 4)$ have been commonly used to achieve the complete reduction of the $\mathrm{Ag}$ salt $(\mathrm{Ag}+)$ to $\mathrm{AgNPs}$ form $\left(\mathrm{Ag}^{\circ}\right)$ (Song, Lee, Park, \& Lee, 2009). Furthermore, chemical compounds, referred to as capping agents, are commonly employed to stabilize the developed nanostructures against aggregation phenomena (de Oliveira \& Cardoso, 2014), (Raveendran et al., 2003). However 
the use of synthetic reducing agent and stabilizing agents is increasingly hampered by costs and secondary toxicity (Abou El-Nour, Eftaiha, Al-Warthan, \& Ammar, 2010) resulting unsuitable for being safely applied in biomedical applications (Rajan, Chandran, Harper, Yun, \& Kalaichelvan, 2015). In recent years, the use of biological methods for the synthesis of metallic nanoparticles has received major attention, since they are usually inexpensive and based on eco-friendly processes (Mohanpuria, Rana, \& Yadav, 2008; Srikar, Giri, Pal, Mishra, \& Upadhyay, 2016; Yong, Beom \& Kim, 2009). Modern synthetic methods are based on the exploitation of natural resources such as whole plants, microorganisms (bacteria and fungi) (Das et al., 2014; Srivastava, Bragança, Ramanan, \& Kowshik, 2013), plant tissues (Ahmed, Ahmad, \& Swami, 2015), fruits (Gnanajobitha et al., 2013; Prathna, Chandrasekaran, Raichur, \& Mukherjee, 2011), plant extracts (Chung, Park, Seung-hyun, Thiruvengadam, \& Rajakumar, 2016) and marine algae (Kumar, Selvi, \& Govindaraju, 2012; Sangeetha \& Saravanan, 2014) which stand out for their inherent biocompatibility and abundant availability (Antony et al., 2013; Prathna et al., 2011; Remya, Rajasree, Aranganathan, \& Suman, 2015; Umashankari, Inbakandan, Ajithkumar, \& Balasubramanian, 2012). Seaweeds represent a promising resource of bioactive molecules, comprising fatty acids, carotenoids, polysaccharides, sterols and oligopeptides which display a wide range of biological activities (Holdt \& Kraan, 2011). Among them sulfated polysaccharides of algal origins have been gaining attention as matrices for biomedical applications thanks to their ascertained bioactivity and chemical versatility. Recently polysaccharides- based extracts obtained from several seaweed species belonging to red, brown and green algae such as Ulva fasciata, Gracilaria birdiae, Turbinaria conoides, Chaetomorpha linum, Laurencia aldingensis, Sargassum siliquosum have been successfully used for the preparation of silver-based nanostructures (El-Rafie, El-Rafie, \& Zahran, 2013; Kannan, Arumugam, Ramya, Manivannan, \& Anantharaman, 2013; Rajeshkumar, Kannan, \& Annadurai, 2013; Vasquez et al., 2016; Vieira, Stein, Andreguetti, Colepicolo, \& Ferreira, 2016). Nevertheless, to the best of our knowledge ulvan, a sulfated polysaccharide extracted from green algae belonging to Ulva sp. (also known as sea lettuce) has never been identified as the active agent in the stabilization and fabrication of AgNPs, being mainly investigated as seaweed extracts in combination with other polysaccharides (Abd, Fatah, El-mongy, \& Eid, 2018; Sangeetha \& Saravanan, 2014; Zohreh, Morteza, Ahmad, \& Akbarzadeh, 2014). In the present study we first explored the activity of ulvan in the reduction process of silver 
ions and the role of the polysaccharide in the stabilization of the obtained AgNPs. Among sulphated polysaccharides of algal origins, ulvan displays unique properties such as unmatched availability and biological activity and specific physicochemical behavior which makes it the most promising candidate for many diverse application (Morelli \& Chiellini, 2010). In particular ulvan availability could be supported through the development of sustainable processes being the relevant resource considered as the most abundant algal biomass worldwide involved in harmful processes for the environment (D'Ayala, Malinconico, \& Laurienzo, 2008). The exploitation of Ulva sp. aimed at obtaining fine chemicals could represent a valuable approach to convert an abundant and cheap biomass, which is commonly referred to as a waste material, into high-value added devices. The chemical composition of ulvan is largely variable and is usually dependent on different factors such as the harvesting season, growth conditions, taxonomic origins and the postcollection treatment of the algal sources (Salima, Benaouda, Noureddine, \& Duclaux, 2013). However, sulfate groups and distinctive sugar monomers such as rhamnose, xylose, glucuronic, and iduronic acid have been identified as the main constituents of ulvan (Robic, Gaillard, Sassi, Leral, \& Lahaye, 2009). The strong antioxidant activity of ulvan, correlated with the distribution and degree of substitution of sulfate groups along the polymeric backbone was found to be responsible of most of displays unique chemical versatility due to the simultaneous presence of hydrophilic and hydrophobic moieties that allow for its use in a wide range of applications.

In the present study ulvan was investigated as active agent in the preparation and stabilization of AgNPs by using simple and eco-friendly methods such as sunlight exposure or heating process. The determination of the stabilizing activity of ulvan toward AgNPs aggregation was investigated by using $\mathrm{NaBH} 4$ as reducing agent in the presence of the polysaccharide. The reducing activity of ulvan was especially explored by using the heating method in absence of chemical additives and physical reducing stimuli, such as sunlight exposure. UV-vis, XRD and TEM analysis were used to evaluate the dimension and size distribution of the inorganic core of the obtained AgNPs while DLS analysis was applied to detect the mean size and size distribution of the colloidal system as a whole, providing additional results about the interaction between ulvan and the inorganic nanoparticles. Optimization of the synthetic experimental parameters was pursued to obtain AgNPs 
without the addition of synthetic reducing and/or capping agents as occurs in the conventional methods.

The most promising developed formulation in terms of stability and size distribution was tested against pathogenic bacteria to evaluate its antimicrobial activity compared to conventional AgNPs obtained by using sodium citrate as reducing and stabilizing agent as reference.

\section{Materials and methods}

\subsection{Materials:}

Ulvan polysaccharide powder extracted from Ulva armoricana was kindly provided by CEVA (Centre d'Etudes et de Valorisation des Algues, Pleubian, France) with an average molecular weight (Mn) 60 kDa (Morelli et al., 2016). Silver nitrate (AgNO3) (purity 99,0\%), sodium borohydride ( $\mathrm{NaBH} 4)$ (98,5\% purity) and sodium citrate dihydrate (purity 99,9\%), Dulbecco's Modified Eagle's Medium (DMEM), Lglutamine, penicillin:streptomycin solution $(10,000 \mathrm{U} / \mathrm{ml}: 10 \mathrm{mg} / \mathrm{ml})$ and Calf bovine serum were purchased from SigmaAldrich and used as received. Plasmocin was obtained from InvivoGen. The murine embryo fibroblast cell line Balb/3T3 Clone A31 was obtained from American Type Culture Collection (ATCC CCL-163). Phosphate Buffer Saline (PBS 10x, 0.1 M) was prepared by dissolving $2.0 \mathrm{~g}$ of $\mathrm{KCl}, 80 \mathrm{~g}$ of $\mathrm{NaCl}, 2.0 \mathrm{~g}$ of $\mathrm{KH} 2 \mathrm{PO} 4 \cdot \mathrm{H} 2 \mathrm{O}$ and $15.6 \mathrm{~g}$ of $\mathrm{Na} 2 \mathrm{HPO} 4 \cdot 12 \mathrm{H} 2 \mathrm{O}$ in $1 \mathrm{~L}$ of deionized water. The $\mathrm{pH}$ of the obtained solution was adjusted to 7.4 with $10 \mathrm{~N}$ $\mathrm{NaOH}$.

\subsection{Synthesis of silver nanoparticles (AgNPs)}

2.2.1 Synthesis of AgNPs in presence of ulvan by using sodium borohydride $\left(\mathrm{NaBH}_{4}\right)$ as reducing agent (UNaB-AgNPs)

In a $250 \mathrm{~mL}$ round bottomed flask equipped with magnetic stirring, a selected amount of ulvan powder (Table 1 ) was added to a solution of $\mathrm{AgNO}_{3}(0.008 \mathrm{~g}$ corresponding to 0.005 $\mathrm{g}$ of $\mathrm{Ag}$ ) in $100 \mathrm{~mL}$ of distilled water. After complete dissolution $5 \mathrm{~mL}$ of an aqueous solution of $\mathrm{NaBH}_{4}(1 \mathrm{mg} / \mathrm{mL})$ was added to the obtained mixture. The solution turned from transparent to light orange after the addition of the reducing agent as a consequence of silver 
salt reduction to AgNPs. The reaction mixture was kept stirring at room temperature until the end of AgNPs formation was detected by UV analysis by monitoring the intensity of the surface plasmon resonance (SPR) bands of silver nanoparticles at regular time intervals. The effect of ulvan concentration was studied by adjusting the ulvan/silver (wt/wt) ratio according to the values reported in Table 1. At the end of the reaction the obtained dispersions were lyophilized $\left(-50^{\circ} \mathrm{C}, 0.07 \mathrm{mbar}\right)$ for $24 \mathrm{~h}$ in order to obtain the dried products for physicochemical characterization.

2.2.2 Synthesis of AgNPs in presence of ulvan under natural sunlight (USL-AgNPs) or artificial sunlight (UAL-AgNPs) irradiation

In a $250 \mathrm{~mL}$ round bottomed flask equipped with magnetic stirring ulvan powder $(0.125 \mathrm{~g})$ was added to a solution of $\mathrm{AgNO}_{3}$ salt (0.002 $\mathrm{g}$ corresponding to $0.001 \mathrm{~g}$ of $\mathrm{Ag}$ ) in $125 \mathrm{~mL}$ of distilled water. After complete dissolution the obtained mixture was kept under natural sunlight irradiation either at $20^{\circ} \mathrm{C}$ or $4{ }^{\circ} \mathrm{C}$ in order to detect the effect of temperature on the reduction process. The reaction mixture was kept under magnetic stirring until the end of AgNPs formation was detected by UV analysis by monitoring the intensity of the surface plasmon resonance (SPR) bands of silver nanoparticles at regular time intervals. A blank experiment was carried out at $20^{\circ} \mathrm{C}$ in completely dark condition. The same procedure was repeated either in basic conditions ( $\mathrm{pH} 11$ ) by adjusting the initial $\mathrm{pH}$ of ulvan solution $(5,5)$ through the addition of $\mathrm{NaOH} 1 \mathrm{M}$ or by using an artificial sunlight lamp as source of irradiation (TRUE-LIGHT, $230 \mathrm{~V} / 50-60 \mathrm{HZ} / 960 \mathrm{LM} / 20 \mathrm{~W}$ ) in order to evaluate respectively the effect of $\mathrm{pH}$ and light origin on the properties of the synthesized AgNPs.

\subsubsection{Synthesis of AgNPs in presence of ulvan by heating method (UHeat-AgNPs)}

In a $250 \mathrm{~mL}$ round bottomed flask equipped with magnetic stirring ulvan powder $(0.062 \mathrm{~g})$ was added to a solution of $\mathrm{AgNO}_{3}$ salt $(0.002 \mathrm{~g}$ corresponding to $0.001 \mathrm{~g}$ of $\mathrm{Ag}$ ) in $125 \mathrm{~mL}$ of distilled water. The effect of ulvan concentration was evaluated by adjusting the ulvan/silver (wt/wt) to 100. The apparatus was covered with aluminum paper to avoid light exposure. The obtained mixtures were kept immersed in an oil bath at $90{ }^{\circ} \mathrm{C}$ under magnetic stirring.

The kinetics of the reactions was assessed by UV-vis spectroscopy by monitoring the intensity of the surface plasmon resonance (SPR) bands of silver nanoparticles at regular 
time intervals. The final solutions were exposed to an excess of $\mathrm{NaBH} 4$ and further analyzed by UV-vis spectroscopy in order to confirm the complete reduction of silver ions occurred by heating. The effect of $\mathrm{pH}$ was investigated by adjusting the initial $\mathrm{pH}$ of the reaction mixtures $(\mathrm{pH}=5,5)$ to 3.0 and 11.0 by using a solution of $\mathrm{HCl}(1 \mathrm{M})$ and $\mathrm{NaOH}(1 \mathrm{M})$ respectively.

All the samples were lyophilized $\left(-50{ }^{\circ} \mathrm{C}, 0.07 \mathrm{mbar}\right)$ for $24 \mathrm{~h}$ in order to obtain the dried products for physicochemical characterization.

\subsubsection{Synthesis AgNPs in presence of citrate by heating method (CitrateHeat-AgNPs)}

In a $250 \mathrm{~mL}$ round bottomed flask equipped with magnetic stirring sodium citrate $(0.500 \mathrm{~g})$ was added to a solution of $\mathrm{AgNO}_{3}$ salt (0.002 $\mathrm{g}$ corresponding to $0.001 \mathrm{~g}$ of $\mathrm{Ag}$ ) in $125 \mathrm{~mL}$ of distilled water. The apparatus was covered with aluminum paper to avoid light exposure. The obtained mixture was kept immersed in an oil bath at $90^{\circ} \mathrm{C}$ under magnetic stirring. The kinetics of the reaction was assessed by UV-vis spectroscopy by monitoring the intensity of the surface plasmon resonance (SPR) bands of silver nanoparticles at regular time intervals.

\subsection{AgNPs Stability}

Stability of UHeat-AgNPs and CitrateHeat-AgNPs were investigated by UV-vis analysis of the relevant dispersions $(0.001 \mathrm{mg} / \mathrm{mL})$ in PBS 1X $(\mathrm{pH}=7.4)$ obtained by dilution of $0,9 \mathrm{~mL}$ of the synthesized AgNPs water dispersions (0.01 mg/mL) with 0,1 mL of PBS 10X ( $\mathrm{pH}=7.4)$. Nanoparticle stability was monitored over time by recording the intensity of the surface plasmon resonance (SPR) bands of silver nanoparticles at regular time intervals. Nanoparticle resistance to oxidation was measured by UV-vis analysis before and after the addition of $0.100 \mathrm{~mL}$ of $30 \% \mathrm{H}_{2} \mathrm{O}_{2}$ to $2,5 \mathrm{~mL}$ silver nanoparticle dispersions obtained at the end of the synthetic processes $(0.01 \mathrm{mg} / \mathrm{mL})$.

\section{$\underline{2.4 \text { Instrumental Methods }}$}

$\mathrm{UV}$-vis spectra were recorded at room temperature in the wavelength region 200-800 nm, by using a Jasco V530 UV-vis spectrophotometer.

Dynamic light scattering (DLS) measurements were carried out by using a Delsa Nano C from Beckman Coulter, Inc. (Fullerton, CA) equipped with a laser diode operating at 658 
nm. Sample dispersions were analyzed in deionized water $(0.5 \mathrm{~mL}, 1 \mathrm{mg} / \mathrm{mL})$ by using a glass cell $\left(0.9 \mathrm{~mL}\right.$ capacity). Scattered light was detected at $165^{\circ}$ angle and analyzed by using a $\log$ correlator over 50 accumulations. The photomultiplier aperture and the attenuator were automatically adjusted to obtain a photon counting rate of ca. $10 \mathrm{kcps}$.

The calculation of the particle size distribution and distribution average were performed by using CONTIN particle size distribution analysis routines using Delsa Nano 3.73 software. The peak average of histograms from intensity, volume and number distributions out of 50 accumulations were reported as the average diameter of the particles.

Zeta potential analyses were carried out by using a Delsa Nano C from Beckman Coulter, Inc. (Fullerton, CA), all samples were analyzed in triplicate at $25^{\circ} \mathrm{C}$, using $3 \mathrm{mM} \mathrm{NaCl} \mathrm{pH}$ 7.4 water solutions as dispersant.

Fourier Transform Infrared Spectroscopy (FTIR) FT-IR spectra were recorded as KBr pellets $(1 / 100 \mathrm{mg})$ in the range $4000-400 \mathrm{~cm}^{-1}$ by using a Jasco FT-IR 410 spectrophotometer with a resolution of $4 \mathrm{~cm}-1$. Each spectrum was recorded after 16 scans.

X-ray diffraction patterns (XRD) were collected using a Rigaku D IIIMAX horizontal-scan powder with a graphite monochromator, operating at $40 \mathrm{kV}$ and $45 \mathrm{~mA}$, and employing nickel-filtered $\mathrm{Cu}$ Ka radiation $(\lambda=0,1542 \mathrm{~nm})$.

Morphological analysis was carried out on a Philips CM12 transmission electron microscope (TEM) with acceleration voltage of 80 and $100 \mathrm{kV}$; sample was prepared by depositing a drop of the AgNPs water suspension on a carbon coated TEM grid and allowing solvent evaporation.

\subsection{Bactericidal activity of UHeat-AgNPs}

The bactericidal activity of UHeat-AgNPs was evaluated against both Gram- (Escherichia coli ATCC 25922 and Pseudomonas aeruginosa ATCC 27853) and Gram+ (Staphylococcus aureus ATCC 33591 and Staphylococcus epidermidis ATCC 35984) bacterial species. Bacteria were grown in Tryptone Soya Broth (TSB) (Oxoid, Basingstoke, UK) until exponential phase and diluted to a final density of $2 \times 10^{6} \mathrm{CFU} / \mathrm{mL}$ in PBS supplemented with $2 \%$ TSB (v/v) (PBS/TSB). A volume of $50 \mu \mathrm{L}$ of the bacterial suspensions was added to $50 \mu$ Lof UHeat-AgNPs at different concentrations (from 0.31 to $5 \mu \mathrm{g} / \mathrm{mL}$ ) and to $50 \mu \mathrm{L}$ of deionized water (viability control). Samples were incubated at $37^{\circ} \mathrm{C}$ with shaking for $24 \mathrm{~h}$, 
serially diluted and plated on Tryptone Soya Agar (TSA) to determine the number of Colony-Forming Units (CFU).

\subsection{Killing kinetics of UHeat-AgNPs and CitrateHeat-AgNPs}

Killing kinetics against E. coli, P. aeruginosa, S. aureus and S. epidermidis were determined using UHeat-AgNPs at their eradicating concentrations. The bactericidal activity of UHeatAgNPs was compared to that exerted by corresponding concentrations of CitrateHeatAgNPs and free ulvan. Ulvan concentrations were established based on the Ulvan/Ag (wt/wt) ratio used for the synthesis of UHeat-AgNPs (Ulvan/Ag=100). Briefly, exponentialphase cultures were diluted in PBS/TSB to a final density of $2 \times 10^{6} \mathrm{CFU} / \mathrm{mL}$. A volume of $50 \mu \mathrm{L}$ of the bacterial suspensions was added to $50 \mu \mathrm{L}$ of UHeat-AgNPs, CitrateHeatAgNPs, ULV or deionized water (viability control). Following different incubation times (1.5, 6 and $24 \mathrm{~h})$, samples were serially diluted and plated on TSA for CFU counting.

\section{$\underline{2.7 \text { Cell culture and cytotoxicity tests }}$}

Murine embryo fibroblast cell line Balb/3T3 Clone A31 was propagated as indicated by the supplier using Dulbecco's Modified Eagle's Medium (DMEM) supplemented with 4mM of L-glutamine, $1 \%$ of penicillin:streptomycin solution $(10,000 \mathrm{U} / \mathrm{ml}: 10 \mathrm{mg} / \mathrm{ml}), 10 \%$ of Calf bovine serum and Plasmocin as antimycotic.

For cytotoxicity tests cells were seeded in 96-well culture plates at a concentration of $2 \times 10^{3} /$ well in a volume of $100 \mu \mathrm{l}$ of medium and incubated at $37^{\circ} \mathrm{C}$ in a $5 \% \mathrm{CO}^{2}$-enriched atmosphere and allowed to proliferate for $24 \mathrm{~h}$ prior to the incubation with samples. The medium from each well was then removed and replaced with medium containing UHeatAgNPs and CitrateHeat-AgNPs at different concentrations of Ag (3.12, 6.25, 12.5, 25, 50 $\mu \mathrm{g} / \mathrm{ml})$. Cells incubated with fresh growth medium were used as blanks. After $24 \mathrm{~h}$ incubation, cells were analyzed for viability with WST-1 tetrazolium salt reagent (Roche Applied Science, Indianapolis, IN, USA). Briefly, cells were incubated for $4 \mathrm{~h}$ with WST-1 reagent diluted $1: 10$, at $37^{\circ} \mathrm{C}$ and $5 \% \mathrm{CO}^{2}$. Measurements of formazan dye absorbance were carried out with a microplate reader (Biorad, Milan, Italy) at $450 \mathrm{~nm}$, using $655 \mathrm{~nm}$ as reference wavelength. Cytotoxicity tests were performed on triplicate for each concentration and data are reported as $\mathrm{IC}_{50}$ (50\% inhibitory concentration, defined as the NPs concentration at which $50 \%$ of cell death in respect to the control is observed) \pm S.D. 


\section{$\underline{\text { 3. Results and Discussion. }}$}

3.1 Synthesis of ulvan-stabilized silver nanoparticles by chemical method (UNaB-AgNPs) AgNPs are conventionally synthesized by chemical reduction of silver ions with $\mathrm{NaBH} 4$ in presence of synthetic stabilizers, through a straightforward and rapid method whose application in biomedical areas has been hampered by toxicity issues. For this reason, green synthesis of metal nanoparticles has been proposed as new eco-friendly alternative to chemical methods in order to reduce the effective process cost and product cytotoxicity. To best of our knowledge ulvan extracted from waste algal biomass belonging to the Ulva sp. has never been employed for the synthesis of AgNPs. In the present work, ulvan was thus investigated for its capability of acting as reducing agent for silver salt to give AgNPs as well as stabilizer of the so formed nanosilver.

At first our attention was focused on assessing Ulvan efficiency as stabilizing agent of AgNPs prepared by using $\mathrm{NaBH}_{4}$ as reducing agent.

The formation of AgNPs in solution was assessed by means of UV-vis absorption spectroscopy through detection of the surface plasmon resonance band (SPR) of AgNPs, which represents a common and easy method to individuate the presence of AgNPs in solution.

SPR analysis represents a valid tool to assess both stability and size of metal nanoparticles since the SPR $\lambda \max$ is commonly affected by metal nanoparticles shape and surface dimension (Noguez, 2007). The SPR bands in the UV-Vis spectra of UNaB-AgNPs synthetized by using different amount of ulvan are reported in the Fig. 1a and the relevant $\lambda \max$ are listed in Table 1 . The presence of a sharp peak in the recorded spectra evidenced the formation of AgNPs with narrow size distribution, highlighting the influence of ulvan in stabilizing the developed colloidal systems. 


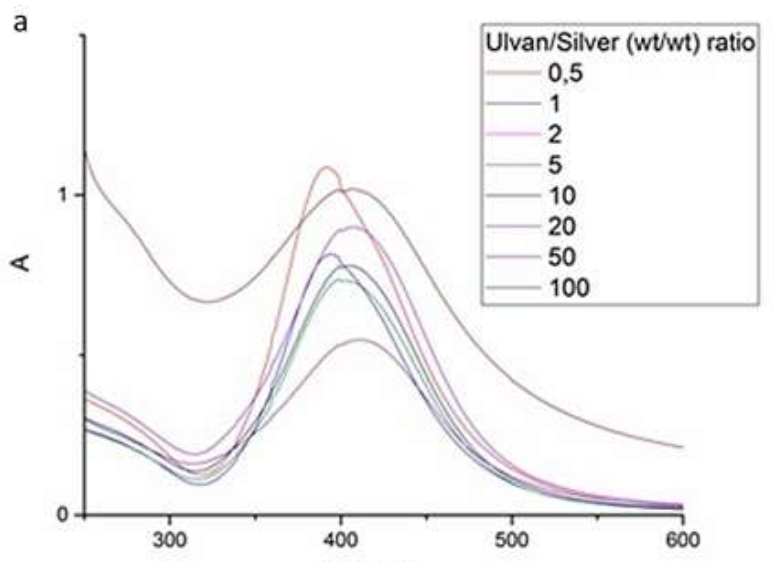

$\lambda(\mathrm{nm})$

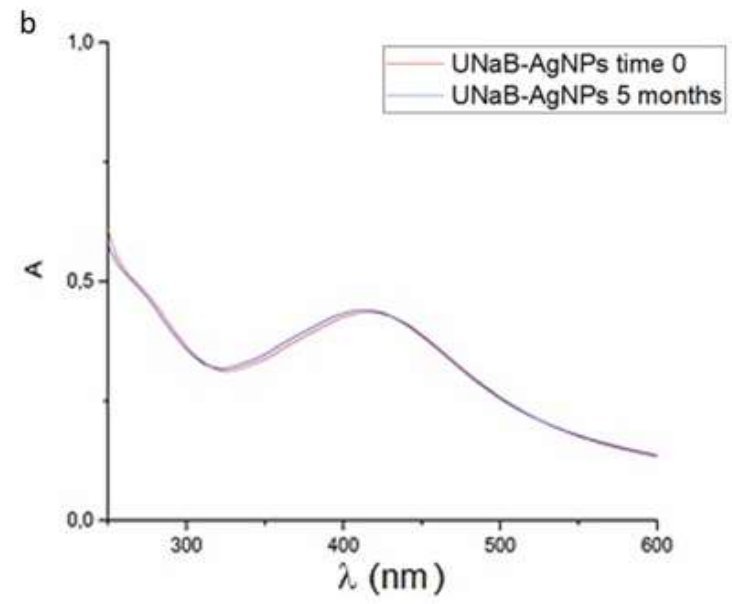

Figure 1. a) UV-vis spectra of AgNPs dispersions obtained by $\mathrm{NaBH}_{4}$ mediated reduction of silver ions in presence of different ulvan/silver weight ratios. b) UV-vis spectra of the sample UNaB-AgNPs (ulvan/silver $(w t / w t)=100)$ at time zero and after 5 months from its preparation.

\begin{tabular}{cc}
\hline $\begin{array}{c}\text { Weight ratio } \\
\text { (ulvan/silver) }\end{array}$ & SPR $\boldsymbol{\lambda}_{\max } \mathbf{( n m )}$ \\
\hline 0,5 & $397 \pm 1,52$ \\
\hline 1 & $397 \pm 1,52$ \\
\hline 2 & $402 \pm 1,00$ \\
\hline 5 & $405 \pm 1,15$ \\
\hline 10 & $408 \pm 1,54$ \\
\hline 20 & $413 \pm 2,64$ \\
\hline 50 & $416 \pm 1,00$ \\
\hline 100 & $416 \pm 0,57$ \\
\hline
\end{tabular}

Table 1. Peak maxima recorded in the UV spectra of UNaB-AgNPs samples synthesized by using different ulvan/ silver (wt/wt) ratios. Mean values out of three replicates are reported. When preparing AgNPs with $\mathrm{NaBH}_{4}$ in absence of ulvan, the obtained nanoparticles were found to aggregate and precipitate from solution.

A red shift of the SPR $\lambda$ max was found to occur according to the amount of ulvan used in the formulations (Table 1). This result suggested a possible contribution of the polysaccharide macromolecules not only as stabilizing agent of the formed nanoparticles, but also to the reduction process of silver ions by $\mathrm{NaBH}_{4}$, since higher $\lambda$ max values are usually related to larger AgNPs dimensions (Berciaud, Cognet, Tamarat, \& Lounis, 2005), (Abdallah, Mohamed, Abdallah, Easawi, \& Negm, 2008). The redshift in the SPR $\lambda \max$ increased as the ratio ulvan/ $\mathrm{Ag}(\mathrm{wt} / \mathrm{wt}$ ) reached a value of 50, above which it was not more 
depending on the amount of polysaccharide used thus indicating a saturation limit for ulvan around the AgNPs core (Table 1).

The stability of the synthetized UNaB-AgNPs was confirmed by UV-vis analysis of the samples stored at room temperature. The intensity and shape of the plasmonic band remained unaffected during storage up to 5 five months (Fig. 1b).

X-ray diffraction analysis (XRD) was carried out to gain deeper information regarding the dimensions of the developed AgNPs. The XRD pattern of UNaB-AgNPs s (ulvan / silver $(w t / w t)=100)$, which represented the nanoparticle system completely saturated by an ulvan outer shell, clearly showed a strong peak at $38.19^{\circ}$ corresponding to the crystal plane of silver nanospheres (111) (Anandalakshmi, Venugobal, \& Ramasamy, 2016). The average crystalline size of the UNaB-AgNPs (ulvan/Ag: 100 (wt/wt) was estimated by using the Debye-Scherrer's equation (Eq. (1)) (Hall, Zanchet, \& Ugarte, 2000):

$$
\mathrm{D}=(\mathrm{k} \lambda) / \beta \cos \theta \quad(\text { Equation } 1)
$$

where $\mathrm{D}$ is the average crystalline size of the NP, $\mathrm{k}$ is geometric spherical factor $(0.9), \lambda$ is the wavelength of X-ray radiation source and $\beta$ is the angular FWHM (full-width at half maximum) of the XRD peak at the diffraction angle $\theta$. By determining the FMWH of the plan (111) Bragg's reflection, the estimated average size of the crystalline nanoparticles (inorganic core) was found to be $33 \mathrm{~nm}$.

The sample UNaB-AgNPs (ulvan / silver (wt/wt)=100) was characterized by DLS analysis in order to evaluate the contribution of the polysaccharide to the dimensions of the colloidal dispersion. Analysis of UNaB-AgNPs (ulvan / silver $(w t / w t)=100)$ revealed the presence of a single population of particles with a mean diameter of $139 \mathrm{~nm}(\mathrm{PI}=0,224)$ thus confirming the presence of the polysaccharide coating around the silver nanoparticle core (Fig. 2a). 

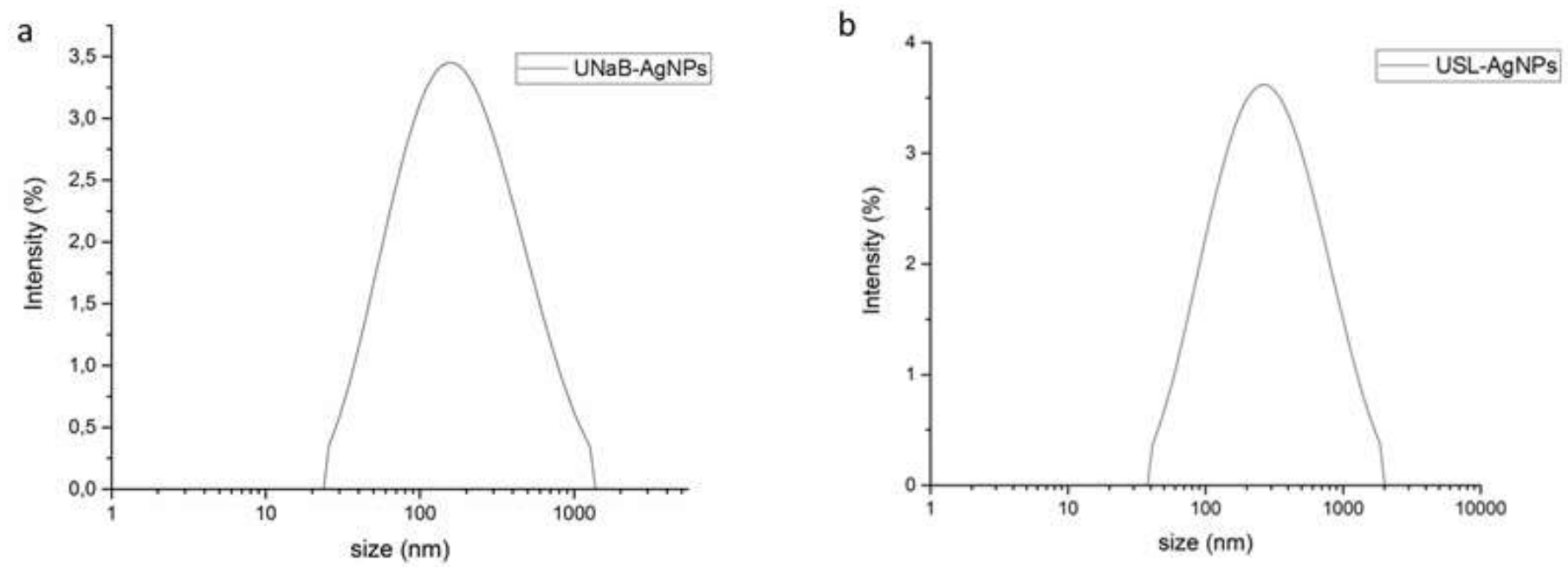

Figure 2: a) DLS size distribution (Intensity) of UNaB-AgNPs (ulvan / silver (wt/wt)=100). b) DLS size distribution of USL-AgNPs $(\mathrm{pH}=11$, Ulvan/ $\mathrm{Ag}(\mathrm{wt} / \mathrm{wt})=100)$ in water by intensity. The SPR bands recorded in the UV-Vis spectra of USL-AgNPs in the mentioned conditions, confirmed the active role of ulvan as reducing/stabilizing agent in the synthesis of the nanoparticles (Fig. 3).

Zeta potential analysis confirmed the synthesized UNaB-AgNPs as highly stable colloidal systems as evidenced by the recorded large negative values $(-41,84 \mathrm{mV})$ provided by the presence of a polyanionicbased shell around the inorganic core.

The current method has evidenced the stabilizing activity and the contribution of ulvan in the formation of AgNPs which could be interpreted by taking into account the abundant presence of hydroxyl, carboxyl and sulphate groups.

\subsection{Synthesis of ulvan-stabilized silver nanoparticles by irradiation method (USL-AgNPs)}

The current method was tested by adopting three different conditions in order to investigate the role of ulvan in AgNPs synthesis and stabilization. The weight ratio of ulvan/Ag used in the experiments was selected as 100 since it was found as the optimal value to create a saturated shell of the polysaccharide around the silver nanoparticle core. In the first experiment (a) the solution containing the silver salt and the polysaccharide was kept under natural sunlight exposure at room temperature at two different $\mathrm{pH}$ of 5,5 and 11 in order to evaluate the effect of the $\mathrm{pH}$ on nanoparticle synthesis; in the second experiment (b) the solution was exposed under natural sunlight irradiation by maintaining it at $4{ }^{\circ} \mathrm{C}$ through immersion in an ice bath in order to evaluate the effect of temperature on nanoparticle 
synthesis; in the last condition (c) the solution was exposed to artificial sunlight at room temperature in order to evaluate the feasibility of carrying out nanoparticle synthesis with in-house laboratory facilities.

a

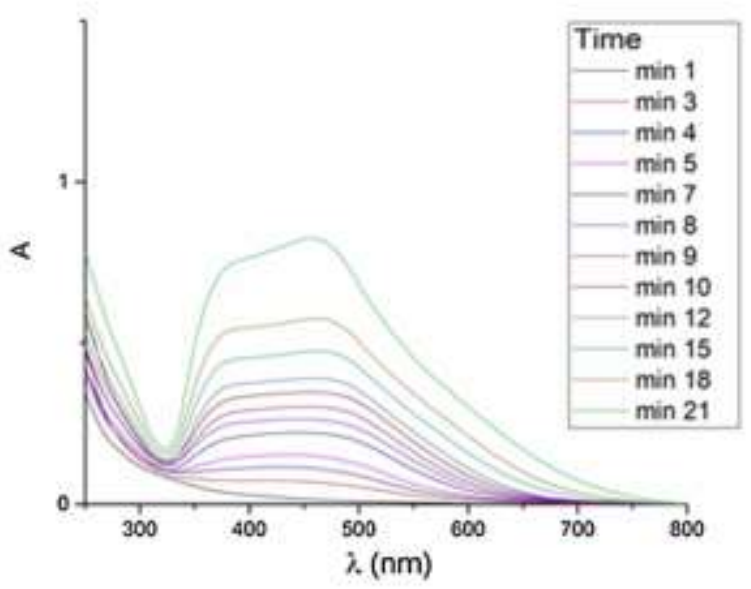

C

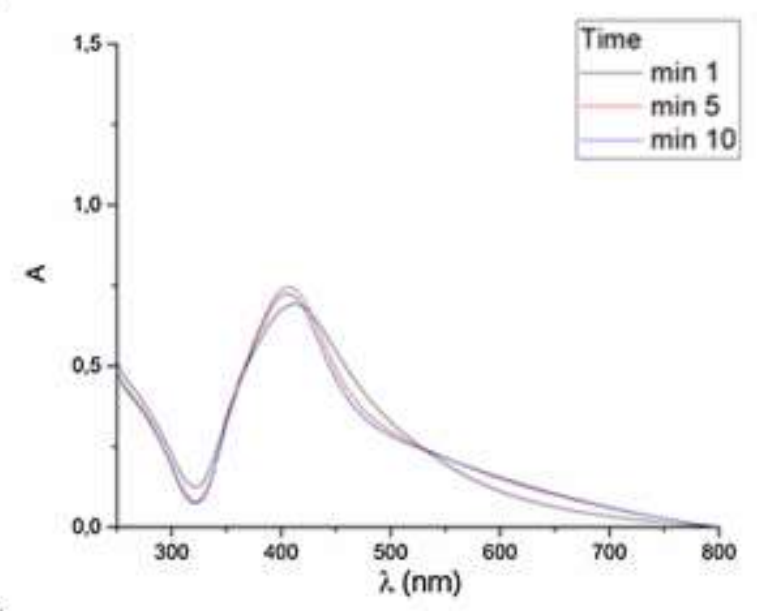

e

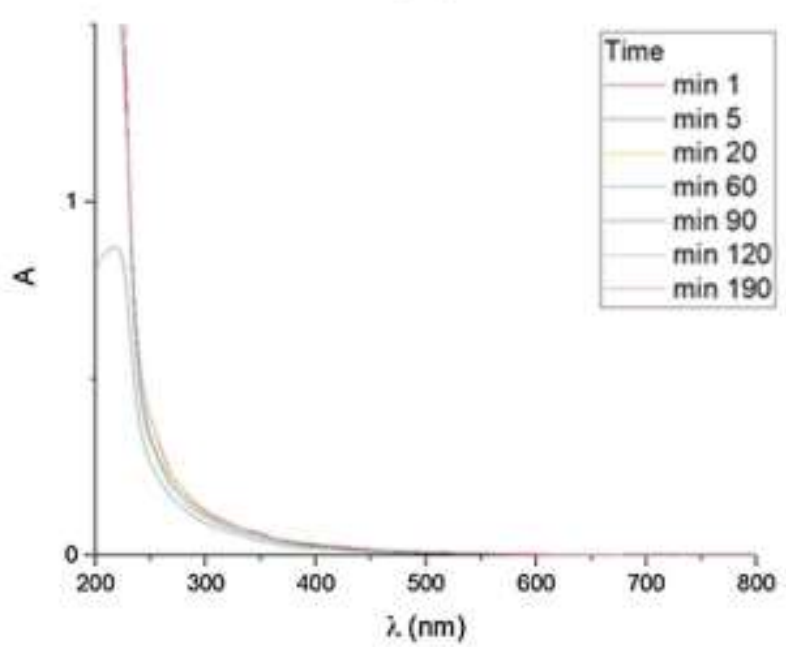

b

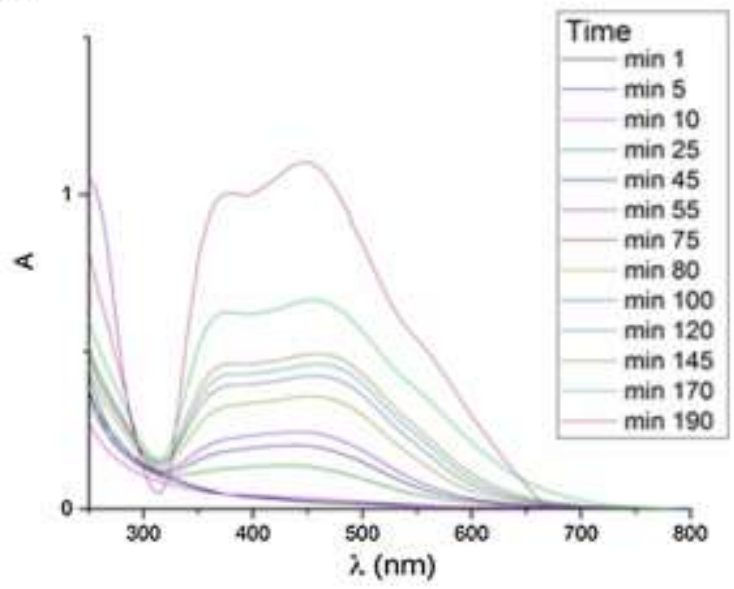

d

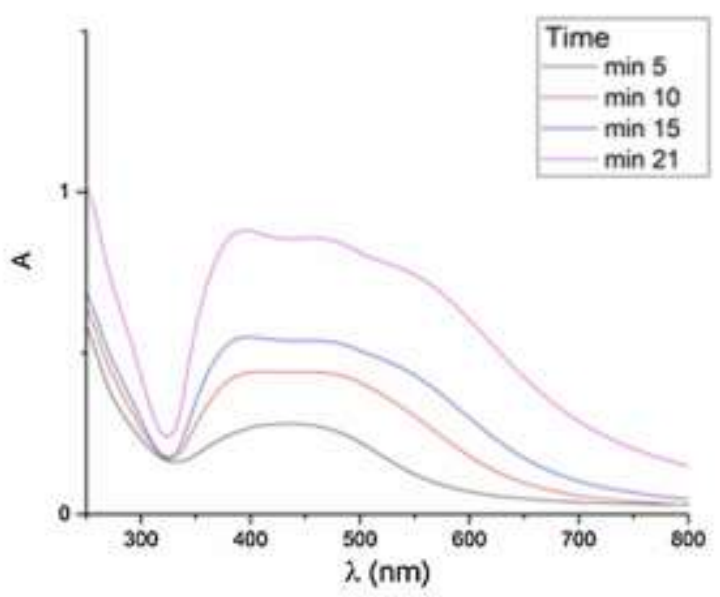

Figure 3: UV-vis spectra of USL-AgNPs synthetized in different conditions: a) under natural sunlight at room temperature at $\mathrm{pH} 5.5 ; \mathrm{b}$ ) under artificial sunlight at room 
temperature at $\mathrm{pH} 5.5$; c) under natural sunlight at room temperature in basic conditions ( $\mathrm{pH} 11)$; d) under natural sunlight at $4{ }^{\circ} \mathrm{C}$ at $\mathrm{pH}$ 5.5; e) in dark conditions.

The adopted experimental conditions led to the formation of colloidal systems characterized by multimodal distribution of dimensions and shapes as evidenced by the broad and complex SPR bands recorded by UV-vis analysis (Qazi \& Javaid, 2016) (Fig. 3). The blank experiments carried out by keeping the solutions in dark conditions did not lead to the formation of AgNPs as evidenced by the lack of discoloration of the analyzed solutions and absence of SPR band in the UV-vis spectra (Fig. 3e).

Natural sunlight exposure carried out in basic conditions led rapidly (5 $\mathrm{min})$ to the formation of a single SPR band indicating the complete reduction of silver salts to AgNPs (Fig. 3c). The exposure of the solutions to natural sunlight in mild acidic conditions (Fig. 3a) resulted in the formation of heterogeneous AgNPs with SPR bands that were superimposable to those obtained with samples exposed to artificial sunlight irradiation at the same $\mathrm{pH}$ (Fig. 3b). However the kinetic of formation of the colloidal systems observed in the two adopted conditions differed significantly, resulting faster in the case of natural sunlight mediated reduction process (Fig. 3a and b). The effect of temperature on AgNPs synthesis under natural sunlight irradiation evidenced the formation of multimodal AgNPs population whose SPR bands were more dispersed at $4{ }^{\circ} \mathrm{C}$ than at room temperature (Fig. $3 \mathrm{~d})$. However the kinetic of the reduction process seemed not to be affected by temperature (Fig. 3a). The synthesized USL-AgNPs dispersions proved to be stable toward aggregation up to 5 months from their preparation notwithstanding the experimental conditions adopted thus indicating the active role of ulvan as stabilizing agent of the developed nanosized colloidal systems.

DLS size analysis revealed the presence of a particle population with a mean size distribution of 215,5 nm (PI 0,312) (Fig. 2b).

The strongly negative Zeta potential value $(-39,7 \mathrm{mV})$ measured for the synthesized USLAgNPs confirmed their high colloidal stability.

Sunlight mediated AgNPs synthesis represents the easiest and most eco-friendly method among those investigated in the present work since it uniquely relies on natural resources of energy for the fabrication of silver nanoparticles. However, it requires a fine optimization 
of the adopted experimental conditions in order to obtain monodispersed nanostructures with reproducible shape and size.

\subsection{Synthesis of ulvan-stabilized silver nanoparticles by heating method (UHeat-AgNPs)}

A further investigated strategy was based on the use of heating to favor the process of reduction of silver ions to AgNPs possibly promoted by ulvan (Hernández-Pinero et al., 2016; Prathna, Raichur, Chandrasekaran, \& Mukherjee, 2013). Solutions of different ulvan/silver (wt/wt) ratios selected over the saturation limit were heated at $90{ }^{\circ} \mathrm{C}$ in dark conditions and analyzed at regular time intervals by UV-vis spectroscopy in order to detect the formation of AgNPs. The effect of the $\mathrm{pH}$ of the solution was evaluated to optimize the kinetic of the reduction process and obtain deeper knowledge on the mechanism of reduction of silver ions by ulvan macromolecules. All the adopted experimental conditions led to the formation of monodisperse AgNPs as evidenced by the sharp surface plasmon resonance peaks recorded by UV-vis spectroscopy (Fig. 4).

a

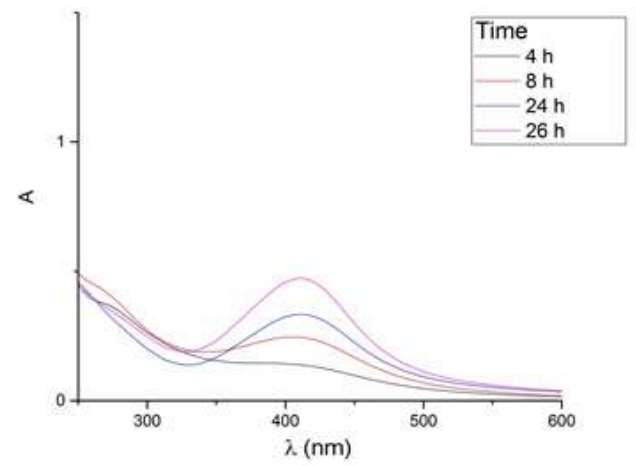

C

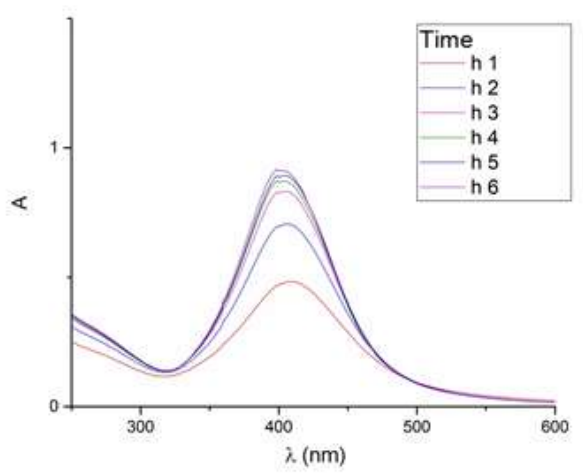

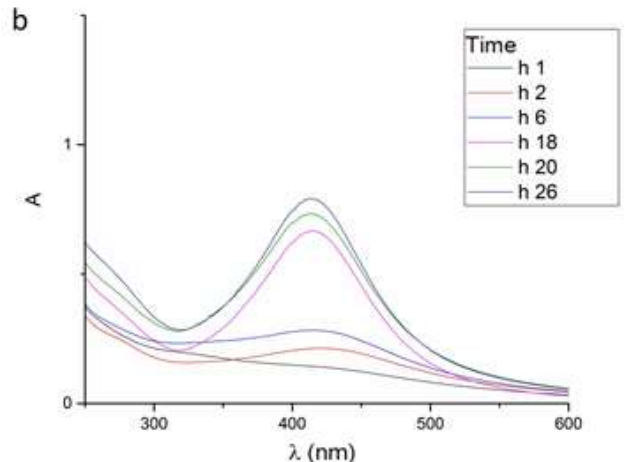

d

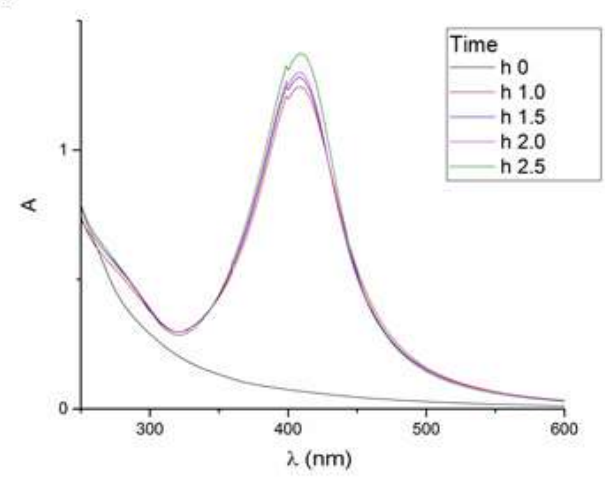

Figure 4: UV-vis spectra of UHeat-AgNPs synthesized in different condition: a) ulvan/silver (wt/wt) ratio of $50(\mathrm{pH}=3.0) ; \mathrm{b})$ ulvan/silver (wt/wt) ratio of $50(\mathrm{pH}=5.5) ; \mathrm{c})$ ulvan/silver $(\mathrm{wt} / \mathrm{wt})$ ratio of $50(\mathrm{pH}=11.0)$; $\mathrm{d})$ ulvan/silver $(\mathrm{wt} / \mathrm{wt})$ ratio of $100(\mathrm{pH}=11.0)$. 
The kinetic of the reduction process was found to be dramatically affected by the $\mathrm{pH}$ of the medium, resulting especially accelerated in basic conditions (Fig. 4c and d). At basic pHs the SPR bands resulted sharp and strong indicating the formation of monomodal AgNPs with high yields of reduction of silver ions (Fig. 4c and d). At $\mathrm{pH} 3$, the reduction process proved to be less effective as evidenced by the presence of SPR bands at lower intensity than those recorded at other $\mathrm{pH}$ values (Fig. 4a). Moreover ulvan concentration was found to strongly affect the kinetic of AgNPs synthesis as emerged from the experiment carried out in basic conditions by using a double amount of polysaccharide thus confirming the active role of ulvan in the reduction process (Fig. $4 \mathrm{~d})$.

The UV-vis analysis of the solution exposed to the most promising conditions ( $\mathrm{pH}=11$, ulvan/Ag $(w t / w t)=100)$ was repeated after addition of a large excess of a strong reductant $\left(\mathrm{NaBH}_{4}\right)$ in order to assess the complete reduction of silver ions by ulvan. The intensity and shape of the SPR band of the synthesized AgNPs were not affected by the addition of $\mathrm{NaBH}_{4}$ indicating that all silver ions were reduced into AgNPs by exposure to ulvan only.

The dimension of the inorganic core estimated by XRD and TEM analysis indicated an average size of the crystalline nanoparticles of $13,3 \mathrm{~nm}$ and $12,5 \mathrm{~nm}$ respectively (Fig. 5a and b).
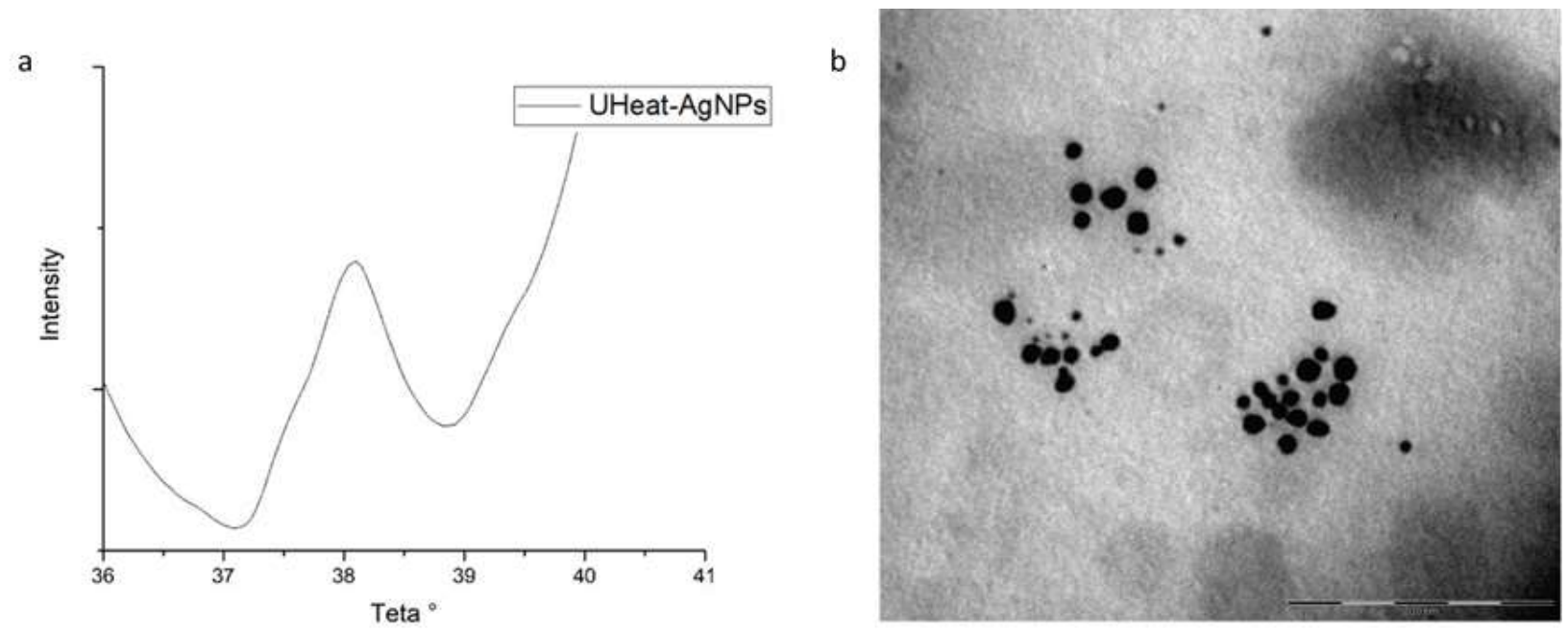

Figure 5: a) XRD pattern of UHeat-AgNPs $(\mathrm{pH}=11$, Ulvan/Ag (wt/wt)=100). b) TEM image of UHeat-AgNPs $(\mathrm{pH}=11$, Ulvan/Ag $(w t / w t)=100)$. DLS size analysis of UHeat-AgNPs prepared adopting the most promising conditions $(\mathrm{pH}=11$, Ulvan/Ag $(w \mathrm{t} / \mathrm{wt})=100)$ revealed the presence of a particle population with monomodal size distribution (mean diameter value 339 nm; PI 0,174) (Fig. 6 a) provided by the presence of an ulvan-based outer 
shell which acted both as reductant and stabilizing agent. Zeta potential analysis confirmed the synthesized UHeat-AgNPs as highly stable colloidal systems as evidenced by the recorded large negative values $(-43,49 \mathrm{mV})$.

TEM image confirmed the size range as estimated by XRD and evidenced the morphological properties of the synthesized UHeat-AgNPs $(\mathrm{pH}=11$, Ulvan/Ag $(w t / w t)=100)$, which were found to adopt a spherical shape (Fig. 5b).

The successful preparation of UHeat-AgNPs carried out in absence of chemical agents and physical stimuli supports the role of ulvan as reductant during the process. Indeed the presence of sulphate groups and reducing end of the polysaccharide chains could be involved in the reduction process of silver ion to AgNPs. To this aim FTIR analysis was used to gain deeper insight onto the activity of the functional groups of ulvan in AgNPs preparation. The comparison among the FTIR spectra of dried UHeat-AgNPs sample and pristine ulvan exposed to the same conditions revealed the presence of a peak at $1380 \mathrm{~cm}-1$ not detected in the spectrum of the native polysaccharide, which was attributed to the shift of the peak corresponding to the bending vibration of the carboxyl group of ulvan involved in the stabilization of AgNPs (Fig. 6b). A new peak recorded at $830 \mathrm{~cm}-1$ emerged from the spectrum of UHeat-AgNPs, which was not detected in the spectrum of the native polysaccharide whose attribution is still uncertain but could be ascribed to the reductant activity of ulvan promoted by sulphate groups (Fig. 6b). Moreover, the presence of peaks at $1305 \mathrm{~cm}^{-1}$ and $1380 \mathrm{~cm}^{-1}$ not detected in the spectrum of native ulvan confirmed the active role of the carboxyl groups of the polysaccharide in the stabilization of the synthesized AgNPs (Fig. 1, Supplementary data).

The current method was selected as model strategy for the synthesis of AgNPs thanks to the absence of chemical additives and high productivity of monomodal silver nanoparticles. 

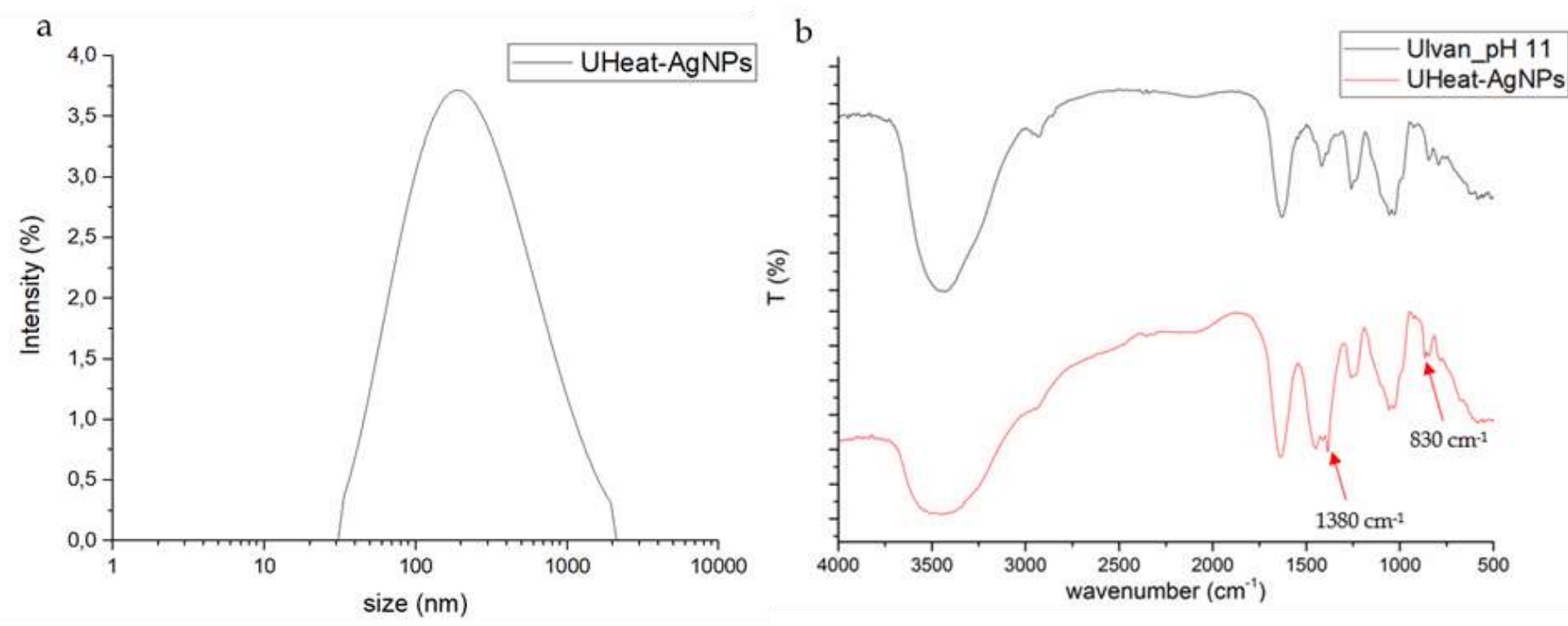

Figure 6: a) DLS size distribution of UHeat-AgNPs $(\mathrm{pH}=11$, Ulvan/Ag $(w t / w t)=100)$ by intensity. b) FT-IR spectra of UHeat-AgNPs ( $\mathrm{pH}=11$, Ulvan/Ag $(w t / w t)=100)$ and pristine ulvan exposed to the same experimental conditions used as a blank.

3.4 Synthesis of citrate-stabilized silver nanoparticles by heating method (CitrateHeatAgNPs)

Conventional AgNPs obtained by using sodium citrate as reductant and stabilizing agent were prepared by heating method and used as reference for antimicrobial activity investigations. The SPR band in the UV-Vis spectra was almost superimposable to that recorded for UHeat-AgNPs indicating that the same shape and dimension of the inorganic core. The DLS size analysis distribution of CitrateHeat-AgNPs revealed the presence of a multimodal size distribution (Mean diameter $36 \mathrm{~nm}, \mathrm{PI}=0,372$ ). The recorded mean hydrodynamic value of UHeat-AgNPs was significantly higher than that of CitrateHeatAgNPs thus supporting the hypothesized presence of a large coating layer of ulvan stabilizing the silver nanoparticles core. Zeta potential analysis of CitrateHeat- AgNPs evidenced the colloidal stability of the synthesized nanoparticles as confirmed by the large negative values recorded $(-37,58 \mathrm{mV})$.

\subsection{AgNPs resistance to oxidation and aggregation}

High stability in biological conditions represents a fundamental requirement that metal nanoparticles formulated for biomedical applications must meet. Indeed metal nanoparticles are prone to aggregation in aqueous environment due to their high surface energy, which cause the complete loss of their physicochemical properties. The presence of 
a stabilizing agent that surrounds the nanostructure can avoid these processes both by electrostatic and steric mechanisms. The most promising sample UHeat-AgNPs $(\mathrm{pH}=11$, Ulvan/Ag $(w t / w t)=100)$ was investigated for its resistance to oxidation and aggregation under physiological conditions by using PBS as investigating medium ( $\mathrm{pH} 7,4 ; 0,01 \mathrm{M})$. UVvis analysis evidenced that UHeat-AgNPs was remarkably more stable than CitrateHeatAgNPs in physiological conditions (Fig. 7). CitrateHeat-AgNPs experienced a modification and a significant reduction of absorbance of the UV-vis spectrum with time due to nanoparticle aggregation and dramatic rearrangement of the colloidal system in presence of salts. AgNPs precipitation occurred as evidenced by discoloration of the suspension and concomitant formation of blackish aggregates. The shape of the UV-vis spectrum of UHeatAgNPs was not affected by incubation of the nanoparticles in PBS indicating their stability in the physiological conditions. Indeed UHeat- AgNPs suspension maintained its original colour upon addition of PBS. The decrease of absorbance recorded for UHeat-AgNPs with time was due to silver nanoparticles precipitation which could be easily reversed by straightforward re-dispersion of precipitate (Fig. 7c). The same operation was not effective in re-dispersion of precipitated CitrateHeat-AgNPs in which irreversible aggregate under physiological conditions were formed (Fig. 7d). The higher stability showed by UHeatAgNPs in respect to CitrateHeat-AgNPs under physiological conditions could be attributed to the presence of a thick ulvan coating surrounding the nanostructured inorganic core, which sterically prevent nanoparticle aggregation. Accordingly ulvan could represent a promising alternative to citric acid as natural stabilizer of AgNPs conventionally used in commercially available formulations. AgNPs resistance to oxidation was studied by adding $\mathrm{H} 2 \mathrm{O} 2$ at the AgNPs suspension in order to predict possible NPs behavior in presence of large amount of reactive oxygen species (ROS).

Fig. 7e and $\mathrm{f}$ confirmed the high stability of both the developed AgNPs during oxidation stress experiment. In this case the presence of steric hindrance seemed not to play a key role for AgNPs resistance towards $\mathrm{H} 2 \mathrm{O} 2$ and ROS species. 
UHeat-AgNPs
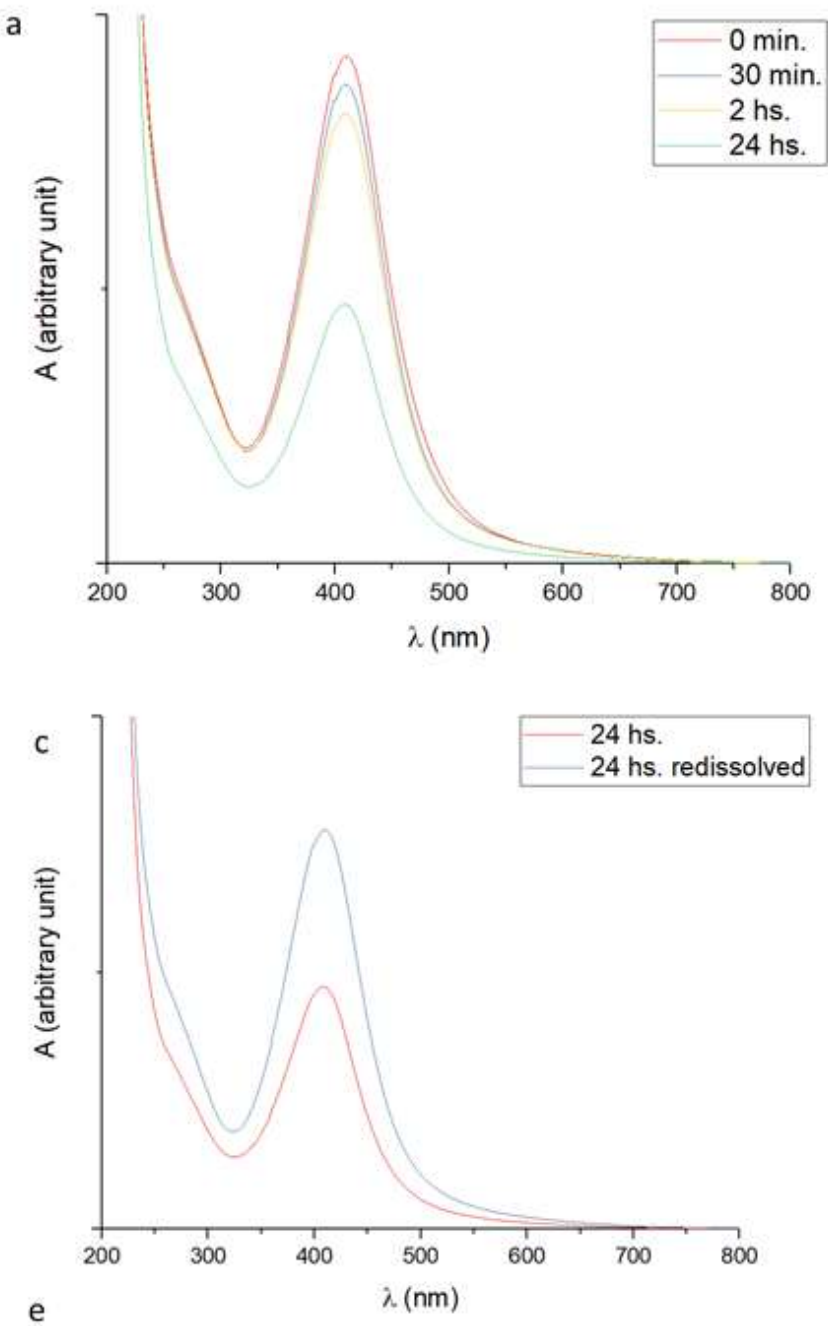

e

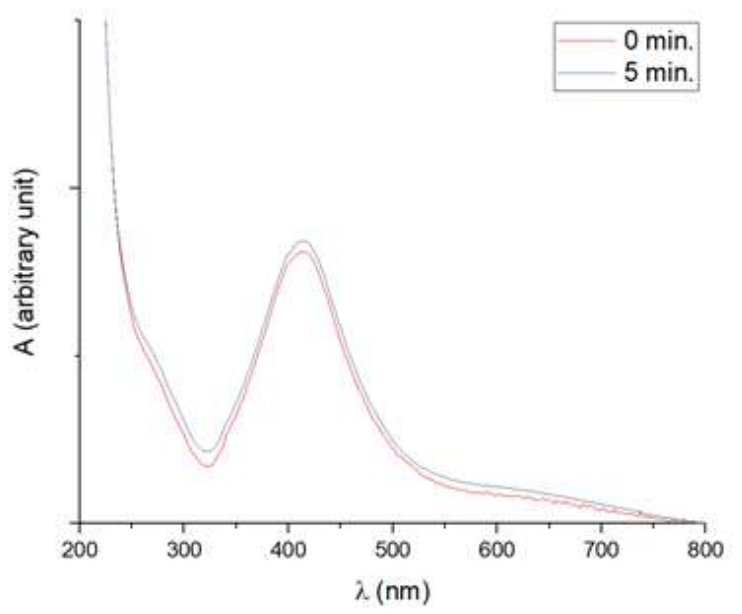

\section{CitrateHeat-AgNPs}
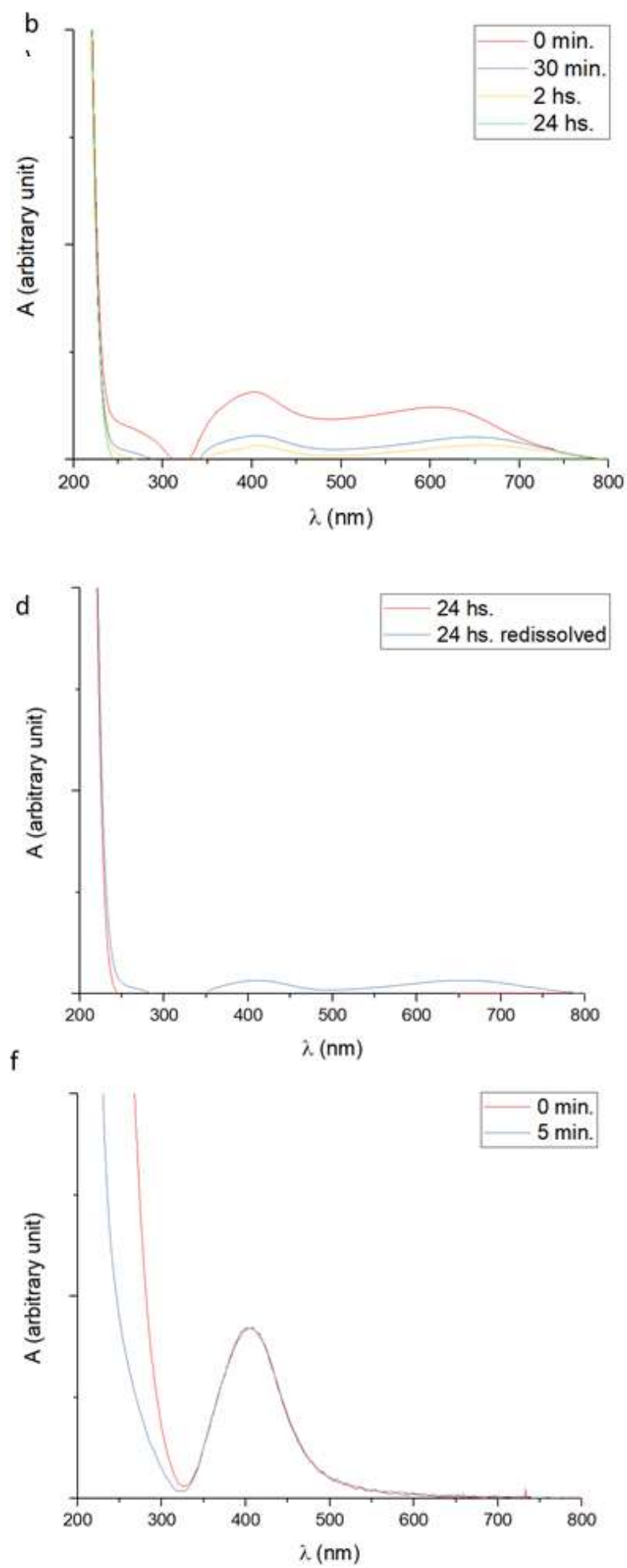

Figure 7: a,b) UV-vis spectra reported in time after the addition of PBS 10X (10\% volume). c,d) UV-vis spectra of resuspension of AgNPs after the addition of PBS 10X (10\% volume). e,f) UV-vis spectra of AgNPs after the addition of $\mathrm{H}_{2} \mathrm{O}_{2}$. 
As shown in Fig. 8, UHeat-AgNPs exerted a marked bactericidal activity against both Gram+and Gram- bacteria resulting in the complete eradication of the initial bacterial inoculum within $24 \mathrm{~h}$ of incubation. A dose-dependent reduction in the number of viable bacteria was observed for Gram+bacteria, whereas the eradicating effect occurred at a threshold dose of UHeat-AgNPs in Gram- bacteria.

\section{$\underline{\text { 3.6 Antibacterial activity of UHeat-AgNPs }}$}

The antibacterial activity of UHeat-AgNPs was evaluated against S. aureus and S. epidermidis as models of Gram+bacteria and against E. coli and P. aeruginosa as models of Gram- bacteria.

For each tested bacterial species, time-kill studies were carried out using eradicating concentrations of UHeat-AgNPs. As shown in Fig. 9, UHeat-AgNPs displayed a more rapid bactericidal effect against Gram- bacteria than against Gram+bacteria. Indeed, in the case of both E. coli and P. aeruginosa (Fig. 9a and b), UHeat-AgNPs determined a reduction of the initial bacterial inoculum to the limit of detection (10 CFU/mL) within $6 \mathrm{~h}$ of incubation. Conversely, UHeat-AgNPs exhibited a bactericidal activity against S. aureus and S. epidermidis (Fig. 9c and d) after $24 \mathrm{~h}$ of incubation. Moreover, UHeat-AgNPs resulted in a stronger antibacterial performance towards all tested bacterial species compared to free ulvan and to CitrateHeat-AgNPs, used as reference system of AgNPs (Fig. 9). Indeed, when tested at equal concentrations, CitrateHeat- AgNPs did not exert any antibacterial effect against Gram-negative bacteria (Fig. 9a and b) and caused only a slight decrease in the initial inoculum in the case of Gram-positive bacteria (1 Log reduction after $24 \mathrm{~h}$ ) (Fig. 9c and d).

Finally a preliminary in vitro cytotoxicity evaluation of UHeat-AgNPs and CitrateHeatAgNPs, was carried out employing the mouse embryo fibroblast cell line Balb3T3. The obtained IC50 values of $10.4 \pm 0.3$ and 10.9 \pm 0.8 for UHeat-AgNPs and CitrateHeat-AgNPs respectively, resulted higher with respect to AgNPs prepared by conventional method (Zou, Feng, Mannerström, Heinonen, \& Pyykkö, 2014). This observation, taking into account that AgNPs prepared and stabilized using Ulvan display a stronger antimicrobial activity with respect to those prepared with citrate, further contribute to support the suitability of the developed UHeat-AgNPs to be employed as antimicrobials for various applications. 


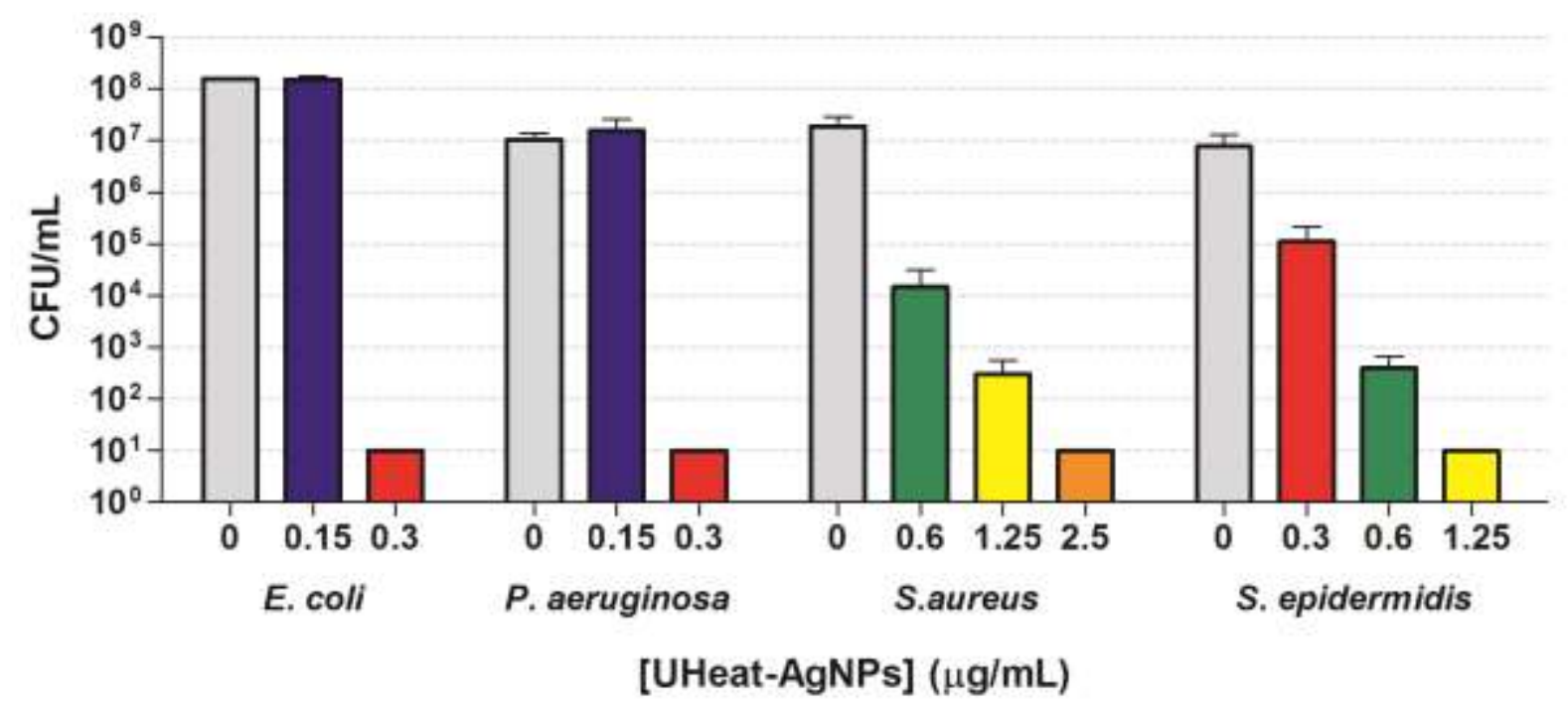

Figure 8: Antibacterial activity of UHeat-AgNPs against E. coli ATCC 25922, P. aeruginosa ATCC 27853, S. aureus ATCC 33591 and S. epidermidis ATCC 35984 after $24 \mathrm{~h}$ of incubation in PBS supplemented with $1 \%$ TSB. A number of $10 \mathrm{CFU} / \mathrm{mL}$ was taken as detection limit. Data are reported as mean \pm standard deviation of at least three independent experiments. 0: untreated control. 
A

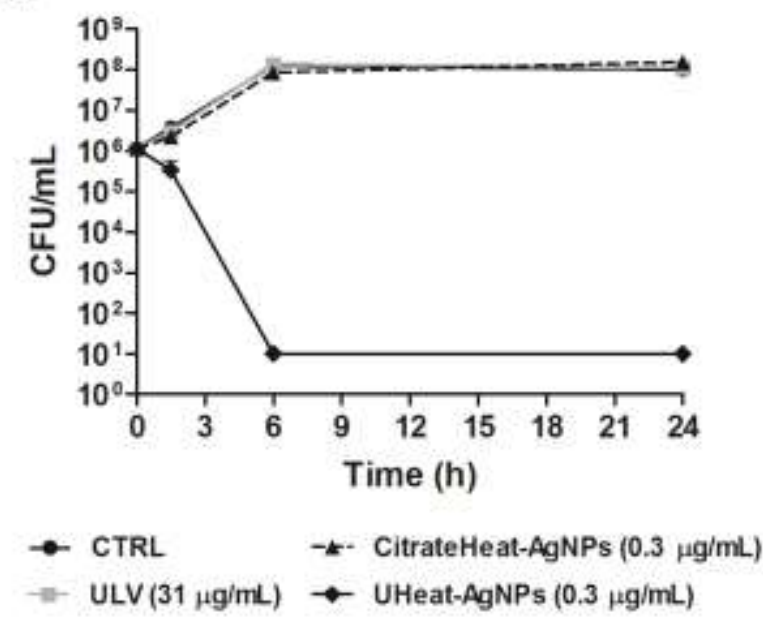

C

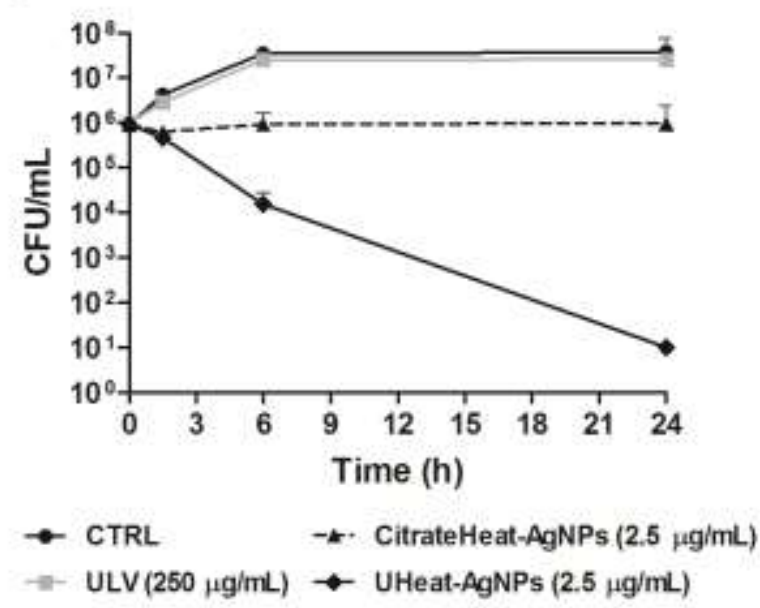

B

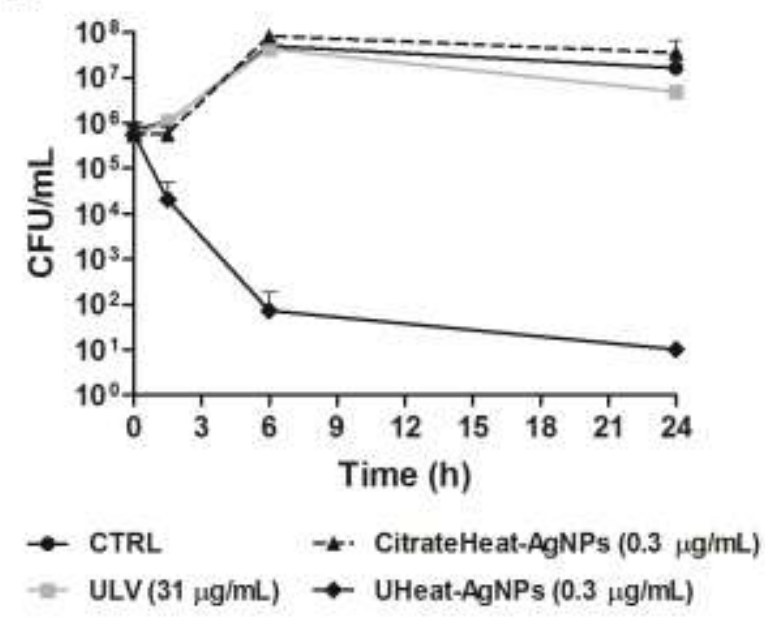

D

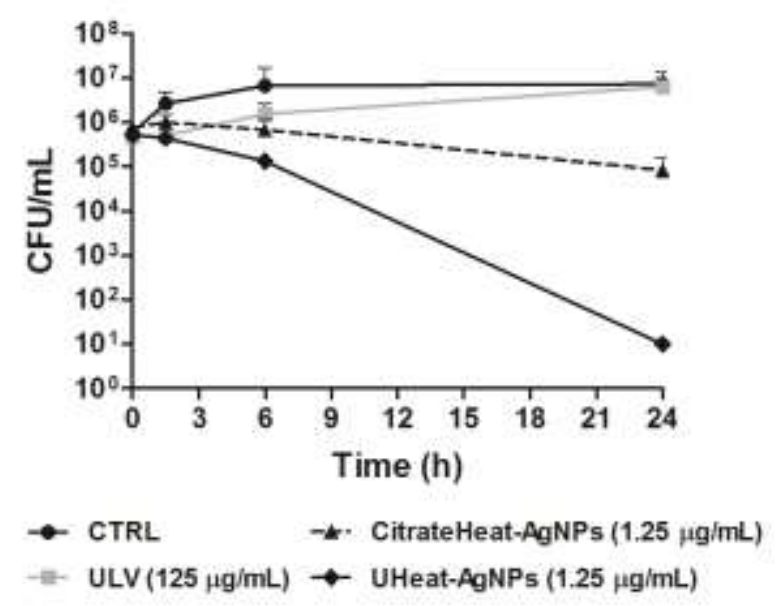

Figure 9: Killing kinetics of UHeat-AgNPs, CitrateHeat-AgNPs and free ulvan (ULV) against E. coli ATCC 25922 (a), P. aeruginosa ATCC 27853 (b), S. aureus ATCC 33591 (c) and S. epidermidis ATCC 35984 (d) in PBS supplemented with 1\% TSB. A number of 10 $\mathrm{CFU} / \mathrm{mL}$ was taken as detection limit. Data are reported as mean \pm standard deviation of at least three independent experiments. CTRL: untreated control. 


\section{CONCLUSIONS:}

In the present manuscript ulvan, a sulphated polysaccharide extracted from green algae belonging to Ulva sp., was unprecedently identified as active compound in the fabrication and stabilization of silver nanoparticles by means of eco-friendly processes. The stability provided by ulvan to the synthesized AgNPs in physiological conditions was significantly higher with respect to traditional stabilizers used in commercially available formulations such as citric acid, due to the formation of a thick polysaccharide shell around the inorganic nanoparticle- based core. The strong and fast antibacterial activity of ulvan stabilized AgNPs against clinically relevant bacterial species of Gram+and Gram- bacteria suggest the use of the algal polysaccharide as an efficient and advanced material for the preparation of antimicrobial compounds for cosmetic and biomedical applications. Collectively an innovative synthetic process to obtain highly stable AgNPs was developed, by exclusively using ulvan as a biocompatible stabilizer and reducing agent with unique biological and physicochemical properties, which can be easily obtained from abundant waste biomass through sustainable processes.

\section{ACKNOWLEDGMENTS:}

The financial support of the University of PisaPRA-2016-50 project entitled 'Functional Materials' and PRA-2017-18 project entitled 'Strategies of diagnosis, prevention, and treatment of medical deviceassociated infections" is gratefully acknowledged. 


\section{References:}

Abd, R., Fatah, E., El-mongy, M. A., \& Eid, K. F. (2018). Research article antibacterial activity of silver nanoparticles using Ulva fasciata extracts as reducing agent and sodium dodecyl sulfate as stabilizer. International Journal of Pharmacology, 14(3), 359-368.

Abdallah, T., Mohamed, M. B., Abdallah, S., Easawi, K., \& Negm, S. (2008). Shape and size dependence of the surface plasmon resonance of gold nanoparticles studied by Photoacoustic technique. The European Physical Journal Special Topics, 364, 361-364.

Abou El-Nour, K. M. M., Eftaiha, A., Al-Warthan, A., \& Ammar, R. A. A. (2010). Synthesis and applications of silver nanoparticles. Arabian Journal of Chemistry, 3(3), 135-140.

Ahmed, S., Ahmad, M., \& Swami, B. L. (2015). Green synthesis of silver nanoparticles using Azadirachta indica aqueous leaf extract. Journal of Radiation Research and Applied Sciences, 9(1), 1-7.

Anandalakshmi, K., Venugobal, J., \& Ramasamy, V. (2016). Characterization of silver nanoparticles by green synthesis method using Pedalium murex leaf extract and their antibacterial activity. Applied Nanoscience, 6(3), 399-408.

Antony, J. J., Ali, M., Sithika, A., Joseph, T. A., Suriyakalaa, U., Sankarganesh, A., et al. (2013). In vivo antitumor activity of biosynthesized silver nanoparticles using Ficus religiosa as a nanofactory in DAL induced mice model. Colloids and Surfaces B, Biointerfaces, 108, 185-190.

Berciaud, S., Cognet, L., Tamarat, P., \& Lounis, B. (2005). Observation of intrinsic size effects in the optical response of individual gold nanoparticles. Nano Letters, 5(3), 515-518.

Carbone, M., Tommasa, D., \& Sabbatella, G. (2016). Silver nanoparticles in polymeric matrices for fresh food packaging. Journal of King Saud University - Science, 28(4), 273279.

Chung, I., Park, I., Seung-hyun, K., Thiruvengadam, M., \& Rajakumar, G. (2016). Plantmediated synthesis of silver nanoparticles: Their characteristic properties and therapeutic applications. Nanoscale Research Letters, 11(40). 
D’Ayala, G. G., Malinconico, M., \& Laurienzo, P. (2008). Marine derived polysaccharides for biomedical applications: Chemical modification approaches. Molecules, 13(9), 2069-2106.

Dakal, T. C., Kumar, A., Majumdar, R. S., \& Yadav, V. (2016). Mechanistic basis of antimicrobial actions of silver nanoparticles. Frontiers in Microbiology, 7, 1831.

Das, V. L., Thomas, R., Varghese, R. T., Soniya, E. V., Mathew, J., \& Radhakrishnan, E. K. (2014). Extracellular synthesis of silver nanoparticles by the Bacillus strain CS 11 isolated from industrialized area. 3 Biotech, 4(2), 121-126.

de Oliveira, J. F. A., \& Cardoso, M. B. (2014). Partial aggregation of silver nanoparticles induced by capping and reducing agents competition. Langmuir, 30(17), 4879-4886.

El-Rafie, H. M., El-Rafie, M. H., \& Zahran, M. K. (2013). Green synthesis of silver nanoparticles using polysaccharides extracted from marine macro algae. Carbohydrate

Polymers, 96(2), 403-410. Franci, G., Falanga, A., Galdiero, S., Palomba, L., Rai, M., Morelli, G., et al. (2015). Silver nanoparticles as potential antibacterial agents. Molecules, 20(5), 8856-8874.

Gajbhiye, S., \& Sakharwade, S. (2016). Silver nanoparticles in cosmetics. Journal of Cosmetics Dermatological Sciences and Applications, 06(01), 48-53.

Ge, L., Li, Q., Wang, M., Ouyang, J., Li, X., \& Xing, M. M. Q. (2014). Nanosilver particles in medical applications: Synthesis, performance, and toxicity. International Journal of Nanomedicine, 16(9), 2399-2407.

Gnanajobitha, G., Paulkumar, K., Vanaja, M., Rajeshkumar, S., Malarkodi, C., Annadurai, G., et al. (2013). Fruit-mediated synthesis of silver nanoparticles using Vitis vinifera and evaluation of their antimicrobial efficacy. Journal of Nanostructure in Chemistry, 3(67).

Hall, B. D., Zanchet, D., \& Ugarte, D. (2000). Estimating nanoparticle size from diffraction measurements. Journal of Applied Crystallography, 33(6), 1335-1341.

Hernández-Pinero, J. L., Terrón-Rebolledo, M., Foroughbakhch, R., Moreno-Limón, S., Melendrez, M. F., Solís-Pomar, F., et al. (2016). Effect of heating rate and plant species on the size and uniformity of silver nanoparticles synthesized using aromatic plant extracts. Applied Nanoscience, 6(8), 1183-1190. 
Holdt, S. L., \& Kraan, S. (2011). Bioactive compounds in seaweed: Functional food applications and legislation. Journal of Applied Phycology, 23(3), 543-597.

Kannan, R. R. R., Arumugam, R., Ramya, D., Manivannan, K., \& Anantharaman, P. (2013). Green synthesis of silver nanoparticles using marine macroalga Chaetomorpha linum. Applied Nanoscience, 3(3), 229-233.

Kim, J. S., Kuk, E., Yu, K. N., Kim, J. H., Park, S. J., Lee, H. J., et al. (2007). Antimicrobial effects of silver nanoparticles. Nanomedicine Nanotechnology Biology and Medicine, 3(1), 95-101.

Kumar, P., Selvi, S. S., \& Govindaraju, M. (2012). Seaweed-mediated biosynthesis of silver nanoparticles using Gracilaria corticata for its antifungal activity against Candida spp. Applied Nanoscience, 3(495-500), 495-500.

Mohanpuria, P., Rana, N. K., \& Yadav, S. K. (2008). Biosynthesis of nanoparticles: Technological concepts and future applications. Journal of Nanoparticle Research, 10(3), 507-517.

Morelli, A., \& Chiellini, F. (2010). Ulvan as a new type of biomaterial from renewable resources: Functionalization and hydrogel preparation. Macromolecular Chemistry and Physics, 211(7), 821-832.

Morelli, A., Betti, M., Puppi, D., Bartoli, C., Gazzarri, M., \& Chiellini, F. (2016). Enzymatically crosslinked ulvan hydrogels as injectable systems for cell delivery. Macromolecular Chemistry and Physics, 217(4), 581-590.

Nguyen, K. C., Seligy, V. L., Massarsky, A., Moon, T. W., Rippstein, P., Tan, J., et al. (2013). Comparison of toxicity of uncoated and coated silver nanoparticles. Journal of Physics Conference Series, 429.

Noguez, C. (2007). Surface plasmons on metal nanoparticles: The influence of shape and physical environment. The Journal of Physical Chemistry C, 111(10), 3606-3619.

Prabhu, S., \& Poulose, E. K. (2012). Silver nanoparticles: Mechanism of antimicrobial action, synthesis, medical applications, and toxicity effects. International Nano Letters, 2(1), 32. 
Prathna, T. C., Chandrasekaran, N., Raichur, A. M., \& Mukherjee, A. (2011). Biomimetic synthesis of silver nanoparticles by Citrus limon (lemon) aqueous extract and theoretical prediction of particle size. Colloids and Surfaces B, Biointerfaces, 82(1), 152-159.

Prathna, T. C., Raichur, A., Chandrasekaran, N., \& Mukherjee, A. (2013). Heat Mediated Synthesis of Silver Nanoparticles Using Citrus limon (Lemon). Asian Journal of Chemistry, 25, 18-20.

Qazi, U. Y., \& Javaid, R. (2016). A review on metal nanostructures: Preparation methods and their potential applications. Advances in Nanoparticles, 5(01), 27-43.

Rajan, R., Chandran, K., Harper, S. L., Yun, S., \& Kalaichelvan, P. T. (2015). Plant extract synthesized silver nanoparticles : An ongoing source of novel biocompatible materials. Industrial Crops and Products, 70, 356-373.

Rajeshkumar, S., Kannan, C., \& Annadurai, G. (2013). Green synthesis of silver nanoparticles using marine brown algae Turbinaria conoides and its antibacterial activity. International Journal of Pharma and Bio Sciences, 3(4), 502-510.

Raveendran, P., Fu, J., \& Wallen, S. L. (2003). Completely “Green" synthesis and stabilization of metal nanoparticles. Journal of the American Chemical Society, 125(46), 13940-13941.

Remya, R. R., Rajasree, S. R. R., Aranganathan, L., \& Suman, T. Y. (2015). An investigation on cytotoxic effect of bioactive AgNPs synthesized using Cassiafistula flower extract on breast cancer cell MCF-7. Biotechnology Reports, 8, 110-115.

Robic, A., Gaillard, C., Sassi, J. F., Leral, Y., \& Lahaye, M. (2009). Ultrastructure of Ulvan: A polysaccharide from green seaweeds. Biopolymers, 91(8), 652-664.

Salima, A., Benaouda, B., Noureddine, B., \& Duclaux, L. (2013). Application of Ulva lactuca and Systoceira stricta algae-based activated carbons to hazardous cationic dyes removal from industrial effluents. Water Research, 47(10), 3375-3388.

Sangeetha, N., \& Saravanan, K. (2014). Biogenic Silver Nanoparticles using marine seaweed (Ulva lactuca) and evaluation of its antibacterial activity. Journal of Nanoscience and Nanotechnology, 2(1), 99-102. 
Song, K. C., Lee, S. M., Park, T. S., \& Lee, B. S. (2009). Preparation of colloidal silver nanoparticles by chemical reduction method. The Korean Journal of Chemical Engineering, 26(1), 153-155.

Srikar, S. K., Giri, D. D., Pal, D. B., Mishra, P. K., \& Upadhyay, S. N. (2016). Green synthesis of silver nanoparticles: A review. Green and Sustainable Chemistry, 06(01), 34-56.

Srivastava, P., Bragança, J., Ramanan, S. R., \& Kowshik, M. (2013). Synthesis of silver nanoparticles using haloarchaeal isolate Halococcus salifodinae BK3. Extremophiles, 17(5), 821-831.

Umashankari, J., Inbakandan, D., Ajithkumar, T. T., \& Balasubramanian (2012). Mangrove plant, Rhizophora mucronata (Lamk, 1804) mediated one pot green synthesis of silver nanoparticles and its antibacterial activity against aquatic pathogens. Aquatic Biosystems, 8(1), 1-7.

Vasquez, R. D., Apostol, J. G., De Leon, J. D., Mariano, J. D., Marie, C., Mirhan, C., et al. (2016). Polysaccharide-mediated green synthesis of silver nanoparticles from Sargassum siliquosum: Assessment of toxicity and hepatoprotective activity. OpenNano, 1, 16-24.

Vieira, A. P., Stein, E. M., Andreguetti, D. X., Colepicolo, P., \& Ferreira, A. M. D. C. (2016). Preparation of silver nanoparticles using aqueous extracts of the red algae Laurencia aldingensis and Laurenciella sp. And their cytotoxic activities. Journal of Applied Phycology, 28(4), 2615-2622.

Wang, L., Hu, C., \& Shao, L. (2017). The antimicrobial activity of nanoparticles: Present situation and prospects for the future. International Journal of Nanomedicine, 12, 12271249.

Yong, J., Beom, S.Æ., \& Kim, S. (2009). Rapid biological synthesis of silver nanoparticles using plant leaf extracts. Bioprocess and Biosystems Engineering, 32(1), 79-84.

Zhang, F., Wu, X., Chen, Y., \& Lin, H. (2009). Application of silver nanoparticles to cotton fabric as an antibacterial textile finish. Fibers and Polymers, 10(4), 496-501.

Zohreh, R., Morteza, Y., Ahmad, N., \& Akbarzadeh, A. (2014). Green Synthesis of Silver Nanoparticles using Ulva flexousa from the Persian Gulf, Iran. Journal of Persian Gulf, 5(15), 9-16. 
Zou, J., Feng, H., Mannerström, M., Heinonen, T., \& Pyykkö, I. (2014). Toxicity of silver nanoparticle in rat ear and BALB/c 3T3 cell line. Journal of Nanobiotechnology, 12, 52. 